\title{
Diagnostic methods based on scattering polarization and the joint action of the Hanle and Zeeman effects
}

\author{
Javier Trujillo Bueno ${ }^{1,2}$ \\ ${ }^{1}$ Instituto de Astrofísica de Canarias, 38205 La Laguna, Tenerife, Spain \\ ${ }^{2}$ Consejo Superior de Investigaciones Científicas (Spain) \\ email: jtb@iac.es
}

\begin{abstract}
Polarized light provides the most reliable source of information at our disposal for diagnosing the physical properties of astrophysical plasmas, including the magnetic fields of the solar atmosphere. The interaction between radiation and hydrogen plus free electrons through Rayleigh and Thomson scattering gives rise to the polarization of the stellar continuous spectrum, which is very sensitive to the medium's thermal and density structure. Anisotropic radiative pumping processes induce population imbalances and quantum coherences among the sublevels of degenerate energy levels (that is, atomic level polarization), which produce polarization in spectral lines without the need of a magnetic field. The Hanle effect caused by the presence of relatively weak magnetic fields modifies the atomic polarization of the upper and lower levels of the spectral lines under consideration, allowing us to detect magnetic fields to which the Zeeman effect is blind. After discussing the physical origin of the polarized radiation in stellar atmospheres, this paper highlights some recent developments in polarized radiation diagnostic methods and a few examples of their application in solar physics.
\end{abstract}

Keywords. Polarization - scattering - Sun: magnetic fields - stars: magnetic fields

\section{Polarization of the stellar continuum radiation}

In the atmospheres of the stars, the dominant contribution to the linear polarization of the visible continuous spectrum comes from scattering at neutral hydrogen in its ground state (Lyman scattering) and Thomson scattering at free electrons (Chandrasekhar 1960). The contribution of these processes to the total absorption coefficient $\left(\chi_{c}\right)$ is quantified by $\sigma_{c}=\sigma_{T} N_{e}+\sigma_{R} n_{1}(H)$, where $\sigma_{T}=6.653 \times 10^{-25} \mathrm{~cm}^{2}$ is the Thomson scattering cross section, $N_{e}$ the electron number density, $n_{1}(H)$ the population of the ground level of hydrogen, and $\sigma_{R}$ the wavelength-dependent Rayleigh cross section. The total absorption coefficient is given by $\chi_{c}=\kappa_{c}+\sigma_{c}$, where $\kappa_{c}$ contains all the relevant non-scattering contributions to the continuum absorption coefficient (e.g., the bound-free transitions in the $\mathrm{H}^{-}$ion). Since at visible wavelengths the continuum absorption coefficient does not depend on the polarization of the incident radiation, the transfer equation for the Stokes parameter $X$ (with $X=I, Q, U$ ) at a given frequency $\nu$ and direction of propagation $\vec{\Omega}$ is given by $\frac{d}{d \tau} X=X-S_{X}$, where $\tau$ (with $d \tau=-\chi_{c} d s$ ) is the monochromatic optical distance along the ray and $S_{X}$ (with $\left.X=I, Q, U\right)$ are the source function components (see Trujillo Bueno \& Shchukina 2009). For example,

$$
\begin{aligned}
S_{Q}= & \frac{\sigma_{c}}{\kappa_{c}+\sigma_{c}}\left\{\frac{3}{2 \sqrt{2}}\left(\mu^{2}-1\right) J_{0}^{2}-\sqrt{3} \mu \sqrt{1-\mu^{2}}\left(\cos \chi \tilde{J}_{1}^{2}+\sin \chi \hat{J}_{1}^{2}\right)\right. \\
& \left.-\frac{\sqrt{3}}{2}\left(1+\mu^{2}\right)\left(\cos 2 \chi \tilde{J}_{2}^{2}+\sin 2 \chi \hat{J}_{2}^{2}\right)\right\}
\end{aligned}
$$



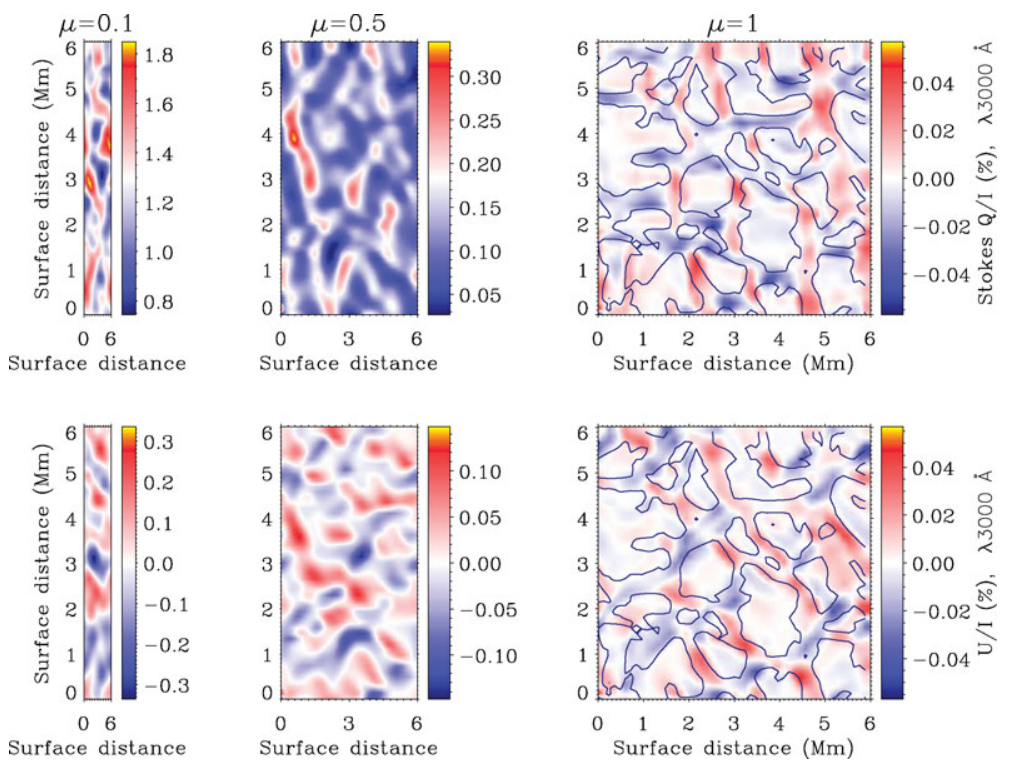

Figure 1. The emergent $Q / I$ (top panels) and $U / I$ (bottom panels) at $3000 \AA$ calculated for three line of sights in a realistic 3D hydrodynamical model of the solar photosphere and accounting for the diffraction limit effect of a 1-m telescope. The reference direction for Stokes $Q$ lies along the vertical direction of the corresponding panel. Note that at $3000 \AA$ the $Q / I$ and $U / I$ images show sizable values (e.g., at $\mu=0.1 Q / I$ varies between $0.8 \%$ and $1.8 \%$, while $U / I$ fluctuates between $\pm 0.3 \%$ ). For more information see Trujillo Bueno \& Shchukina (2009).

and

$$
S_{U}=\frac{\sigma_{c}}{\kappa_{c}+\sigma_{c}} \sqrt{3}\left\{\sqrt{1-\mu^{2}}\left(\sin \chi \tilde{J}_{1}^{2}-\cos \chi \hat{J}_{1}^{2}\right)+\mu\left(\sin 2 \chi \tilde{J}_{2}^{2}-\cos 2 \chi \hat{J}_{2}^{2}\right)\right\},
$$

where the orientation of the ray is specified by $\mu=\cos \theta$ (with $\theta$ the polar angle) and by the azimuthal angle $\chi$. In these source function expressions the $J_{P}^{K}$ quantities (with $K=0,2$ and $P=0,1,2)$ are the spherical components of the radiation field tensor (see $\S 5.11$ in Landi Degl'Innocenti \& Landolfi 2004), which quantify the symmetry properties of the radiation field at the spatial point under consideration. Thus, $J_{0}^{0}$ is the familiar mean intensity, $J_{0}^{2}$ quantifies its anisotropy, while the real and imaginary parts of $J_{P}^{2}$ (with $P=1,2$ ) (i.e., $\tilde{J}_{P}^{2}$ and $\hat{J}_{P}^{2}$, respectively) measure the breaking of the axial symmetry. Obviously, $J_{1}^{2}$ and $J_{2}^{2}$ are zero in a plane-parallel or spherically symmetric model atmosphere, but they can have significant positive and negative values in a 3D model of stellar surface convection (see figure 3 of Trujillo Bueno \& Shchukina 2009). Therefore, Eqs. (1.1) and (1.2) tell us that $U=0$ in $1 \mathrm{D}$ models and that the key observational signatures of the symmetry breaking effects in a $3 \mathrm{D}$ model (caused by its horizontal atmospheric inhomogeneities) are non-zero $U$ signals at any on-disk position and non-zero Stokes $Q$ and $U$ signals for the line of sight (LOS) with $\mu=1$, which corresponds to forward-scattering geometry.

Figure 1 shows the $Q / I$ and $U / I$ images that we would see at $3000 \AA$ if we could observe the solar continuum polarization at very high spatial and temporal resolution. Obviously, without spatial and/or temporal resolution $U / I=0$ and the only observable quantity would be $Q / I$, whose wavelength variation at a solar disk position close to the limb has been determined semi-empirically by Stenflo (2005). 


\section{Polarization of the spectral line radiation}

\subsection{The Zeeman effect}

The spectral line polarization produced by the Zeeman effect is caused by the wavelength shifts between the $\pi\left(\Delta M=M_{u}-M_{l}=0\right)$ and $\sigma_{b, r}(\Delta M= \pm 1)$ transitions, whose wavelength positions and strengths should (in general) be calculated within the framework of the Paschen-Back effect theory. Such wavelength shifts are of course due to the presence of a magnetic field, which causes the atomic and molecular energy levels to split into different magnetic sublevels characterized by their magnetic quantum number $M$ (e.g., Stenflo 1994; Landi Degl'Innocenti \& Landolfi 2004).

The Zeeman effect is most sensitive in circular polarization (quantified by the Stokes $V$ parameter), with a magnitude that for not too strong fields scales with the ratio, $\mathcal{R}$, between the Zeeman splitting and the Doppler broadened line width (which is usually very much larger than the natural width of the atomic levels!), and in such a way that the emergent Stokes $V(\lambda)$ profile changes its sign for opposite orientations of the magnetic field vector. This so-called longitudinal Zeeman effect responds to the line-of-sight component of the magnetic field. In contrast, the transverse Zeeman effect responds to the component of the magnetic field perpendicular to the line of sight, so that the linear polarization Stokes $Q$ and $U$ profiles change sign when the direction of the transverse component changes by $\pm 90^{\circ}$. Given that for not too strong fields the Stokes $Q$ and $U$ signals produced by the transverse Zeeman effect scale as $\mathcal{R}^{2}$, their amplitudes are normally below the noise level of present observational possibilities for intrinsically weak fields (typically $\mathrm{B} \lesssim 100$ gauss in solar spectropolarimetry). A good new is that the mere detection of the Zeeman effect polarization signature(s) implies the presence of a magnetic field. One disadvantage of the polarization of the Zeeman effect as a diagnostic tool is that it is blind to magnetic fields that are tangled on scales too small to be resolved.

\subsection{Anisotropic radiation pumping and atomic level polarization}

The illumination of the atoms in a stellar atmosphere is anisotropic. This is easy to understand if we consider the case of a plasma structure embedded in the optically thin outer layers of a stellar atmosphere (e.g., a solar coronal filament), because the incident radiation comes mainly from the underlying quiet photosphere and is contained within a cone of half aperture $\alpha \leqslant 90^{\circ}$, with the vertex centered on the point under consideration. The larger the height above the visible stellar "surface" the smaller $\alpha$ and the larger the anisotropy factor $w=\sqrt{2} \mathcal{A}=\sqrt{2} J_{0}^{2} / J_{0}^{0}$, where $J_{0}^{0}$ is the familiar mean intensity and $J_{0}^{2} \approx \oint \frac{\mathrm{d} \vec{\Omega}}{4 \pi} \frac{1}{2 \sqrt{2}}\left(3 \mu^{2}-1\right) I_{\nu, \vec{\Omega}}$. Neglecting the $\vec{\Omega}$ dependence of the incident intensity $I_{\nu, \vec{\Omega}}$, and assuming that it is unpolarized, it is easy to find that $w=[1+\cos \alpha] \cos \alpha / 2$, which shows that in this case where the $\vec{\Omega}$-dependence of $I_{\nu, \vec{\Omega}}$ is neglected $0 \leqslant w \leqslant 1$, with $w=1$ for the limiting case of a unidirectional unpolarized light beam that propagates along the vertical direction. The radiation field is also anisotropic within a stellar atmosphere itself (i.e., at heights where the overlying atmospheric plasma is not optically thin), but in this case $w$ can be positive or negative. As shown in the right panel of Fig. 2, at such heights the outgoing radiation shows limb darkening (i.e., it is predominantly vertical) while the incoming radiation shows limb brightening (i.e., it is predominantly horizontal). Therefore, there is competition, because "vertical" rays (i.e., with $|\mu|>1 / \sqrt{3}$ ) make positive contributions to $w$, while "horizontal" rays (i.e., with $|\mu|<1 / \sqrt{3}$ ) make negative contributions to $w$. It wins the subset of intensities (outgoing or incoming) having the largest variation with $\mu$. Figure 4 in the review paper by Trujillo Bueno (2001) shows how is $\mathcal{A}=J_{0}^{2} / J_{0}^{0}$ within a Milne-Eddington model atmosphere for increasing values of its source function gradient. 

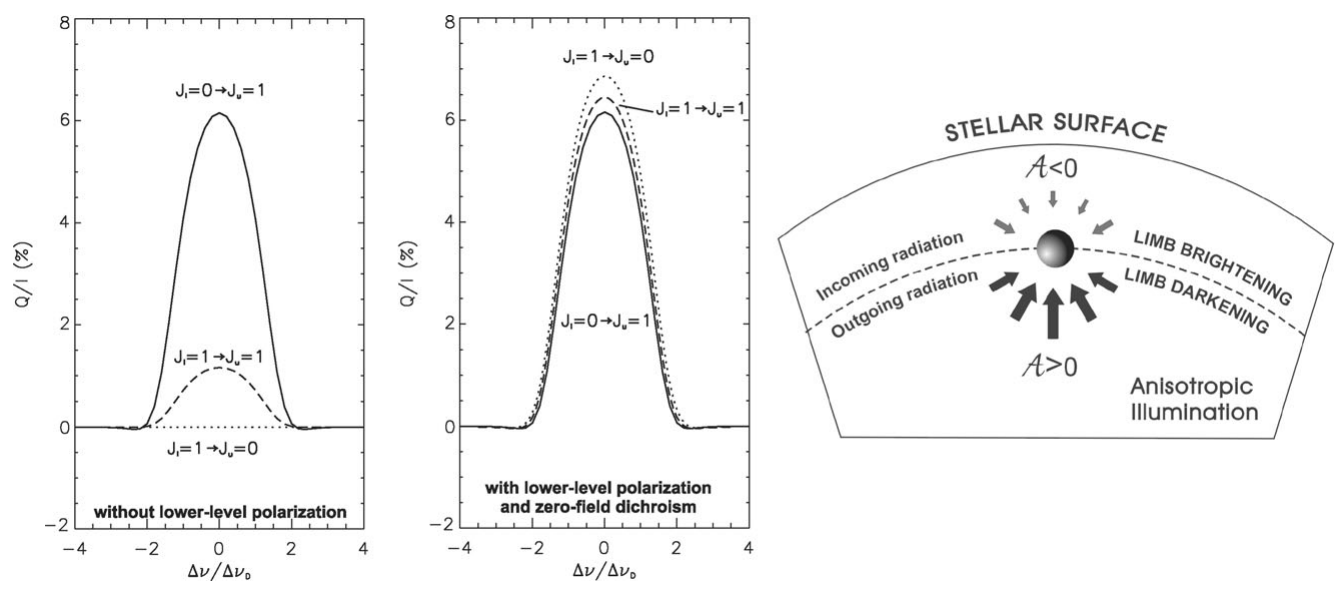

Figure 2. The emergent $Q / I$ profiles (for a LOS with $\mu=0.1$ ) of three line transitions calculated in a model atmosphere with $T=6000 \mathrm{~K}$ and $B=0 \mathrm{G}$. All these $Q / I$ signals are solely due to the atomic level polarization that results from the anisotropic illumination illustrated in the right panel. Left panel: assuming that the lower level is unpolarized. Middle panel: taking into account the full impact of lower-level polarization. Like in figures 3 and 4 below, the reference direction for Stokes $Q$ is the parallel to the closest stellar limb. From Trujillo Bueno (1999).

Why do we worry about the anisotropy of the radiation field? The reason lies in that anisotropic radiation pumping processes in a stellar atmosphere tend to induce population imbalances among the magnetic sublevels of the atomic levels (i.e., atomic level polarization), in such a way that the populations of substates with different values of $|M|$ are different. There are two key mechanisms capable of producing directly atomic level polarization through the absorption of anisotropic radiation (Happer 1972; Trujillo Bueno 2001): upper-level selective population pumping (which occurs when some substates of the upper level have more chances of being populated than others) and lower-level selective depopulation pumping (which occurs when some substates of the lower level have more chances of being depopulated than others).

Why do we worry about the atomic level polarization? The reason lies in that spectral line polarization can be produced by the mere presence of atomic level polarization, i.e., by the existence of population imbalances among the sublevels pertaining to the upper and/or lower atomic levels of the line transition under consideration. Upper-level polarization produces selective emission of polarization components (i.e., the emitted light is polarized, even in the absence of a magnetic field), while lower-level polarization causes selective absorption of polarization components or "zero-field" dichroism (i.e., the transmitted beam is polarized, even in the absence of a magnetic field).

A useful expression to estimate the amplitude of the emergent fractional linear polarization is the following generalization of the Eddington-Barbier formula (Trujillo Bueno 2001), which establishes that the emergent $Q / I$ at the line center of a sufficiently strong spectral line when observing along a line of sight specified by $\mu=\cos \theta$ is approximately given by

$$
Q / I \approx \frac{3}{2 \sqrt{2}}\left(1-\mu^{2}\right)\left[\mathcal{W} \sigma_{0}^{2}\left(J_{u}\right)-\mathcal{Z} \sigma_{0}^{2}\left(J_{l}\right)\right]
$$

where $\mathcal{W}$ and $\mathcal{Z}$ are numerical factors that depend on the angular momentum values $(J)$ of the lower $(l)$ and upper $(u)$ levels of the transition (e.g., $\mathcal{W}=1$ and $\mathcal{Z}=0$ for a line with $J_{l}=0$ and $J_{u}=1, \mathcal{W}=0$ and $\mathcal{Z}=1$ for a transition with $J_{l}=1$ and $J_{u}=0$, and 
$\mathcal{W}=\mathcal{Z}=-1 / 2$ for a line with $J_{l}=J_{u}=1$ ), while $\sigma_{0}^{2}=\rho_{0}^{2} / \rho_{0}^{0}$ quantifies the fractional atomic alignment of the upper or lower level of the line transition under consideration $\dagger$

Consider the three line transitions of Fig. 2, and the corresponding emergent $Q / I$ profiles obtained by solving numerically the scattering polarization problem in an unmagnetized model atmosphere assuming a two-level atomic model for each line independently. The left panel corresponds to calculations carried out assuming that the lower level is completely unpolarized, while the middle panel takes into account the full impact of lower-level polarization. Note that when the atomic polarization of the lower level is taken into account then the "null line" (i.e., that with $J_{l}=1$ and $J_{u}=0$ ) shows the largest $Q / I$ amplitude. In conclusion, "zero-field" dichroism is a very efficient mechanism for producing linear polarization in the spectral lines that originate in a stellar atmosphere.

\subsection{The Hanle effect}

In order to understand what the Hanle effect is it is first necessary to clarify that in the general case where polarization phenomena are taken into account, the full description of an atomic system requires to specify, for each $J$-level, a density matrix with $(2 J+$ $1)^{2}$ elements. The diagonal ones, $\rho_{J}(M, M)$, quantify the populations of the individual sublevels and the non-diagonal ones, $\rho_{J}\left(M, M^{\prime}\right)$, the quantum coherences between each pair of them. We say that the quantum coherence $\rho_{J}\left(M, M^{\prime}\right)$ is non-zero when the wave function presents a well defined phase relationship between the pure quantum states $|J M\rangle$ and $\left|J M^{\prime}\right\rangle$. The law of transformation of the density-matrix under a rotation of the reference system chosen for the specification of its elements indicates that it is actually very common to find non-zero coherences when describing the excitation state of an atomic system under the influence of anisotropic radiative pumping (e.g., Landi Degl'Innocenti \& Landolfi 2004).

Consider a reference system whose $z$-axis (the quantization direction of total angular momentum) is chosen along the direction of the applied magnetic field and $J$-levels whose sublevels are not affected by possible crossings and/or repulsions with the sublevels pertaining to other levels. In this simplest case, the Hanle effect tends to reduce and dephase the quantum coherences with respect to the non-magnetic case, without modifying the population imbalances. For the Hanle effect to operate the magnetic field must be inclined with respect to the symmetry axis of the pumping radiation field. What happens is that as the sublevels are split by the magnetic field, the degeneracy of the $J$-level under consideration is lifted and the coherences are modified. This gives rise to a characteristic magnetic-field dependence of the linear polarization of the emergent spectral line radiation that provides an attractive diagnostic tool of magnetic fields in astrophysics.

Approximately, the amplitude of the emergent spectral line polarization is sensitive to magnetic strengths between $0.1 B_{H}$ and $10 B_{H}$, where the critical Hanle field intensity $\left(B_{H}\right.$, in gauss) is that for which the Zeeman splitting of the $J$-level under consideration is similar to its natural width: $B_{\mathrm{H}}=\left(1.137 \times 10^{-7}\right) /\left(t_{\text {life }} g_{J}\right)$ (with $g_{J}$ the level's Landé factor and $t_{\text {life }}$ its radiative lifetime in seconds). If the line's lower level is the ground level or a metastable level, its $t_{\text {life }}\left(J_{l}\right) \approx 1 / B_{l u} J_{0}^{0}$ (with $J_{0}^{0}$ the mean intensity of the spectral line radiation and $B_{l u}$ the Einstien coefficient for the absorption process), which for relatively strong solar spectral lines is typically between a factor $10^{2}$ and $10^{3}$ larger than the upper-level lifetime $\left(t_{\text {life }}\left(J_{u}\right) \approx 1 / A_{u l}\right.$, where $A_{u l}$ is Einstein's coefficient for the spontaneous emission process). For this reason, in solar-like atmospheres the lower-level

$\dagger$ For example, $\rho_{0}^{0}(J=1)=\left(N_{1}+N_{0}+N_{-1}\right) / \sqrt{3}$ and $\rho_{0}^{2}(J=1)=\left(N_{1}-2 N_{0}+N_{-1}\right) / \sqrt{6}$, where $N_{1}, N_{0}$ and $N_{-1}$ are the populations of the magnetic sublevels. 
Hanle effect is normally sensitive to magnetic fields in the milligauss range, while the upper-level Hanle effect is sensitive to fields in the gauss range.

Typically, in $90^{\circ}$ scattering geometry (e.g., when observing off the solar limb) the largest polarization occurs for the unmagnetized case, with the direction of the linear polarization perpendicular to the scattering plane. In the presence of a magnetic field pointing either towards the observer (case $a$ ) or away from him/her (case $b$ ) the polarization amplitude is significantly reduced with respect to the previously discussed unmagnetized case. Moreover, the direction of the linear polarization is rotated with respect to the zero field case. Normally, this rotation is counterclockwise for case (a) and clockwise for case (b). Therefore, when opposite magnetic polarities coexist within the spatio-temporal resolution element of the observation the direction of the linear polarization is like in the unmagnetized reference case, simply because the rotation effect cancels out. However, the polarization amplitude is indeed reduced with respect to the zero field reference case, which provides an "observable" that can be used to obtain information on hidden, tangled magnetic fields at subresolution scales in the solar atmosphere (Stenflo 1994; Trujillo Bueno et al. 2004).

On the other hand, in forward scattering geometry (e.g., when the line of sight points to the solar disk center) we have zero polarization in the absence of magnetic fields, while non-zero linear polarization in the presence of an inclined field. The ensuing $Q / I$ amplitude reaches its maximum possible value for a magnetic strength such that the ensuing Zeeman splitting is much larger than the level's natural width (i.e., for $B>10 B_{H}$, approximately). In forward scattering geometry the linear polarization is created by the Hanle effect, a physical phenomenon that has been demonstrated via spectropolarimetry of solar coronal filaments in the He I $10830 \AA$ multiplet (Trujillo Bueno et al. 2002).

\section{Diagnostic methods based on the continuum polarization}

As $§ 1$ suggests, without spatial resolution the fractional linear polarization of the stellar continuum radiation is largely determined by the effective polarizability, $\sigma_{c} /\left(\kappa_{c}+\sigma_{c}\right)$, and by the radiation field's anisotropy, $\mathcal{A}=J_{0}^{2} / J_{0}^{0}$, which in turn depend on the density and thermal structure of the stellar atmosphere under consideration. Collisional and/or magnetic depolarization do not play any role on the polarization of the continuum radiation of the Sun's visible spectrum. Therefore, the more realistic is the thermal and density structure of a given solar atmospheric model, the closer to the empirical data will be the calculated linear polarization of the solar continuum radiation. Interestingly, Fig. 3 demonstrates that 3D radiative transfer modeling of the polarization of the Sun's continuous spectrum in a well-known 3D hydrodynamical model of the solar photosphere shows a notable agreement with Stenflo's (2005) semi-empirical data, significantly better than that obtained via the use of $1 \mathrm{D}$ atmospheric models.

\section{Diagnostic methods based on the spectral line polarization}

\subsection{The scattering polarization of the $C a$ II IR triplet}

A suitable diagnostic window for investigating the thermal and magnetic structure of the "quiet" solar chromosphere is the scattering polarization in the Ca II IR triplet (see Manso Sainz \& Trujillo Bueno 2003a, 2009). The radiative transfer modeling results of Fig. 4 indicate that while the scattering polarization in the $8498 \AA$ line shows a strong sensitivity to inclined magnetic fields with strengths between $1 \mathrm{mG}$ and $10 \mathrm{G}$, the emergent linear polarization in the $8542 \AA$ and $8662 \AA$ lines is sensitive to magnetic fields in the milligauss 

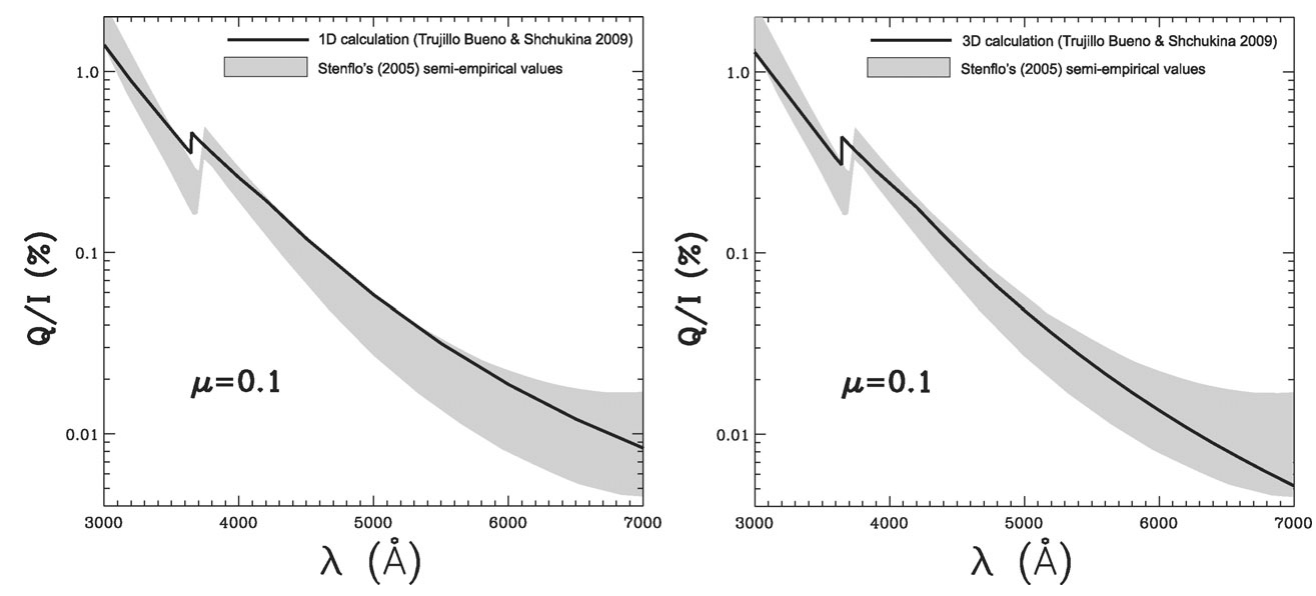

Figure 3. The wavelength variation of the polarization of the Sun's continuous spectrum. The gray shaded areas corresponds to Stenflo's (2005) semi-empirical data. The solid lines show the results of radiative transfer calculations in a well-known 1D semi-empirical model (left panel) and in a well-known 3D hydrodynamical model (right panel).

range. The reason for this very interesting behavior is that the scattering polarization in the $8498 \AA$ line gets a significant contribution from the selective emission processes that result from the atomic polarization of the short-lived upper level, while that in the 8542 $\AA$ and $8662 \AA$ lines is dominated by the selective absorption processes that result from the atomic polarization of the metastable (long-lived) lower levels. Therefore, in "quiet" regions of a stellar atmosphere the magnetic sensitivity of the linear polarization of the $8542 \AA$ and $8662 \AA$ lines is controled by the lower-level Hanle effect, which implies that in regions with $1 \lesssim B \lesssim 50 \mathrm{G}$ the Stokes $Q$ and $U$ profiles are only sensitive to the orientation of the magnetic field vector. In such regions the $8498 \AA$ line is however sensitive to both the orientation and the strength of the magnetic field through the upper-level Hanle effect. In summary, "zero-field" dichroism is the key physical origin of the enigmatic scattering polarization observed by Stenflo et al. (2000) in the Ca II IR triplet (see Manso Sainz \& Trujillo Bueno 2003a). Interestingly, this linear dichroism caused by the mere presence of population imbalances in the lower levels of the line transitions may also be operating in other astrophysical objects (e.g., supernovae) and should be taken into account when interpreting spectropolarimetric observations in other spectral lines besides the Ca II IR triplet itself.

\subsection{The Hanle and Zeeman effects in the He I $10830 \AA$ multiplet}

A suitable diagnostic tool for determining the magnetic field of plasma structures embedded in the solar chromosphere and corona (e.g., spicules, prominences and filaments) can be achieved by observing and interpreting the polarization signals produced by the joint action of atomic level polarization and the Hanle and Zeeman effects in the He I $10830 \AA$ triplet. This multiplet originates between the metastable state, $2^{3} \mathrm{~S}_{1}$, and the first excited term, $2^{3} \mathrm{P}_{2,1,0}$, of the triplet system of He I. Therefore, its blue component has $J_{l}=1$ and $J_{u}=0$ (i.e., it is a "null" line), while its red blended components have $J_{u}=2$ and $J_{u}=1$.

For field strengths $B \lesssim 100 \mathrm{G}$ the linear polarization of the He I $10830 \AA$ triplet is fully dominated by the atomic level polarization that is produced by anisotropic radiation pumping processes. Since the blue component has $J_{u}=0$ its linear polarization can only 

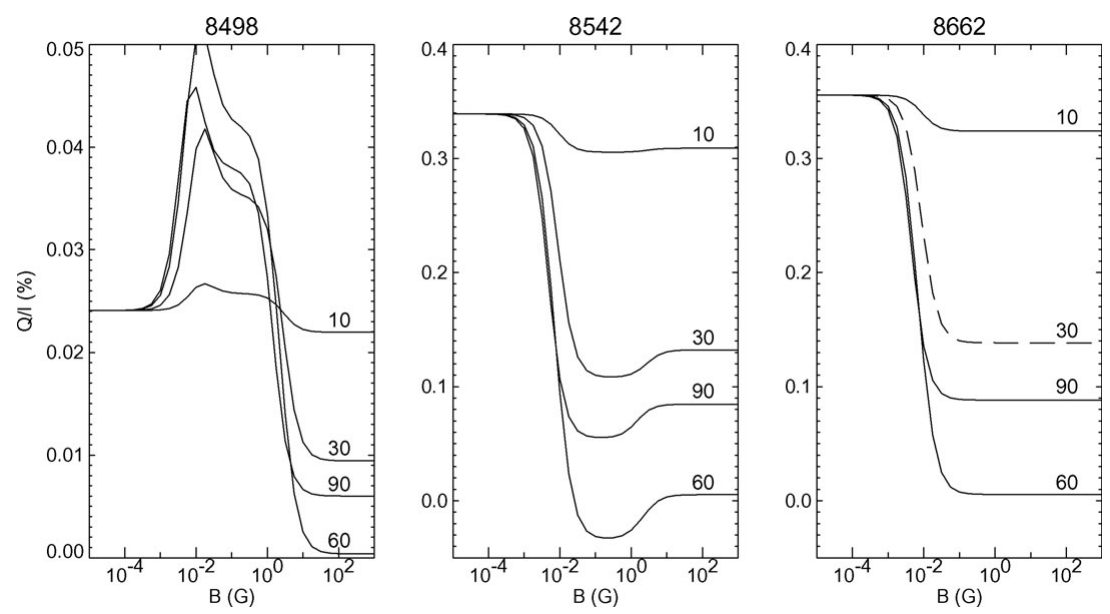

Figure 4. The emergent fractional linear polarization of the Ca II IR triplet calculated for a LOS with $\mu=0.1$ in a semi-empirical model of the solar atmosphere. Each curve corresponds to the indicated inclination, with respect to the solar local vertical direction, of the assumed random-azimuth magnetic field. From Manso Sainz \& Trujillo Bueno (2009).

be due to selective absorption of polarization components (i.e., "zero-field" dichroism), so that its detection requires to observe a light beam after it has passed through an optically pumped plasma (e.g., when observing a solar coronal filament against the bright background of the solar disk). The left panels of Fig. 5 show an example of the Stokes $I$ and $Q$ profiles of a solar coronal filament observed in forward scattering geometry at the solar disk center. The detection of linear polarization in the blue line implies that the metastable state, $2^{3} \mathrm{~S}_{1}$, was significantly polarized. Even more interesting is the conclusion that in the forward scattering geometry of this observation the mere detection of linear polarization in any of the components of the He I $10830 \AA$ triplet implies the presence of a magnetic field inclined with respect to the solar radius vector through the observed point (Trujillo Bueno et al. 2002).

Another example of a "weakly" magnetized plasma (i.e., with $B \lesssim 100 \mathrm{G}$ ) is that of the dynamic jets that we call spicules, needle-shaped plasma structures emanating from the solar network regions and reaching heights as large as $10000 \mathrm{~km}$ in the chromosphere. The determination of the magnetic field that channels the spicular motions can be achieved by modeling observations of the polarization of the He I $10830 \AA$ and/or $5876 \AA$ (or $\mathrm{D}_{3}$ ) multiplets in $90^{\circ}$ scattering geometry at various heights above the visible solar limb (see Centeno et al. 2009; and more references therein). The right panels of Fig. 5 show an example of one of our most recent spectropolarimetric observations in the He I $10830 \AA$ triplet. In this $90^{\circ}$ scattering geometry observation the linear polarization of the scattered light provides direct information only about the polarization of the excited states. For this reason, we do not see now any linear polarization signal in the blue line $\left(J_{l}=1\right.$ and $J_{u}=0$ ), in spite of the fact that its lower level is probably polarized. However, we do see linear polarization in the red blended component, which implies that its upper levels are polarized. Interestingly, the observed non-zero Stokes $U$ signal is produced by the Hanle rotation of the direction of linear polarization, which indicates the presence of a magnetic field inclined with respect to the local vertical direction. The presence of a magnetic field is also indicated by the observed Stokes $V$ profile, which in the He I 10830 $\AA$ triplet is caused by the longitudinal Zeeman effect. Its detection is indeed crucial for the determination of the full magnetic field vector via theoretical modeling based on the 

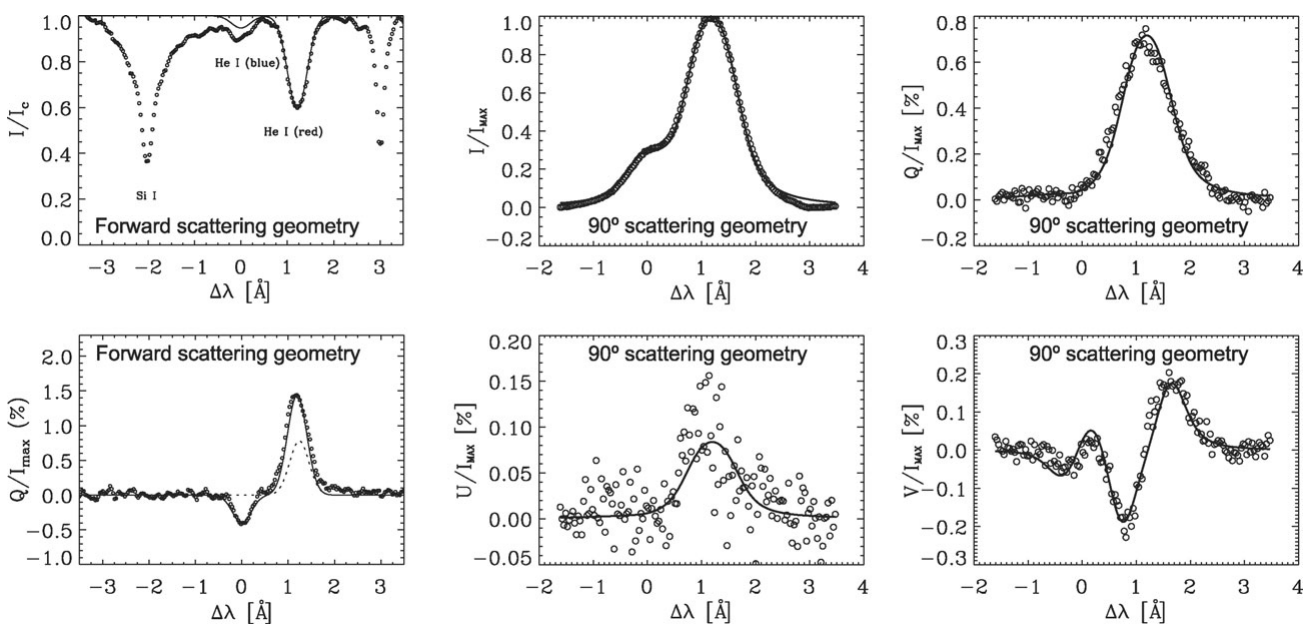

Figure 5. Forward scattering panels (from Trujillo Bueno et al. 2002): The open circles show the $I$ and $Q$ profiles of the He I $10830 \AA$ triplet observed in a coronal filament at the solar disk center. The solid lines show the theoretical profiles corresponding to a magnetic field with $B=20 \mathrm{G}$ and inclination $\theta_{B}=105^{\circ}$. The dotted line shows the theoretical Stokes $Q$ profile when the lower level is assumed to be unpolarized. The positive direction of Stokes $Q$ is parallel to the projection of the magnetic field vector on the solar surface. $90^{\circ}$ scattering panels (from Centeno et al. 2009): The open circles show the Stokes profiles observed in spicules of the very quiet Sun. The solid lines show the theoretical profiles corresponding to $B \approx 36 \mathrm{G}, \theta_{B} \approx 38^{\circ}$ and azimuth $\chi_{B} \approx-2^{\circ}$. The Stokes $Q$ reference direction is the parallel to the observed solar limb.

quantum theory of polarization (see the solid lines fit), because for magnetic strengths larger than only a few gauss the He I $10830 \AA$ multiplet is in the saturation regime of the upper-level Hanle effect $\left(B>10 B_{H} \approx 8 \mathrm{G}\right)$, where the linear polarization signals are only sensitive to the inclination and azimuth of the magnetic field.

For field strengths $100<B \lesssim 2000 \mathrm{G}$ the linear polarization of the He I $10830 \AA$ triplet is caused by the joint action of atomic level polarization and the transverse Zeeman effect (see Fig. 2 of Trujillo Bueno \& Asensio Ramos 2007). As shown by these authors, the impact of atomic level polarization on the linear polarization of the emergent radiation in the He I $10830 \AA$ multiplet can indeed be very significant, even for magnetic field strengths as large as $1000 \mathrm{G}$. Finally, for field strengths $B>2000 \mathrm{G}$ the linear polarization of the He I $10830 \AA$ triplet turns out to be dominated by the transverse Zeeman effect.

\subsection{Some computer programs for modeling the Hanle and Zeeman effects}

In order to carry out the above-mentioned type of investigations in an efficient way, it is useful and important to have at our disposal reliable codes for the synthesis and/or inversion of Stokes profiles caused by atomic level polarization and the Hanle and Zeeman effects. To this end, we have developed MULTIPOL (Manso Sainz \& Trujillo Bueno 2003b) and HAZEL (Asensio Ramos et al. 2008), which are based on the quantum theory of spectral line polarization described in the monograph by Landi Degl'Innocenti \& Landolfi (2004). The multilevel radiative transfer calculations of Fig. 4 were carried out with MULTIPOL, while the four right panels of Fig. 5 show an example of the application of the inversion option of HAZEL to spectropolarimetric observations of solar spicules in the He I $10830 \AA$ triplet. HAZEL (from HAnle and ZEman Light) is a public, userfriendly computer program for the synthesis and inversion of Stokes profiles caused by the joint action of atomic level polarization and the Hanle and Zeeman effects in plasma structures embedded in a stellar atmosphere. 


\section{Concluding comment}

One of the greatest challenges in astrophysics is the exploration of cosmical magnetic fields (e.g., in the solar corona, in circumstellar envelopes, in acreting systems, etc.) The physical mechanisms I have discussed here (anisotropic radiative pumping, atomic level polarization, "zero-field" dichroism, and the Hanle and Zeeman effects) operate in many astrophysical systems, not only in the solar atmosphere. In particular, the spectral line polarization caused by atomic level polarization and its modification by the Hanle effect provides key information, impossible to obtain via conventional spectropolarimetry.

\section{Acknowledgements}

Finantial support by the Spanish Ministry of Science through project AYA2007-63881 and by the European Commission via the SOLAIRE network is gratefully acknowledged.

\section{References}

Asensio Ramos, A., Trujillo Bueno, J., \& Landi Degl'Innocenti, E. 2008, ApJ 683, 542 Centeno, R., Trujillo Bueno, J., \& Asensio Ramos, A. 2009, ApJ, in preparation Chandrasekhar, S. 1960, Radiative Transfer, Dover Publications, Inc.

Happer, W. 1972, Rev. Mod. Phys. 44, 169

Landi Degl'Innocenti, E. \& Landolfi, M. 2004, Polarization in Spectral Lines, Kluwer

Manso Sainz, R. \& Trujillo Bueno, J. 2003a, Phys. Rev. Lett. 91, 111102

Manso Sainz, R. \& Trujillo Bueno, J. 2003b, in ASP Conf. Ser. Vol. 307, Solar Polarization, ed. J. Trujillo Bueno \& J. Sánchez Almeida, 251

Manso Sainz, R. \& Trujillo Bueno, J. 2009, ApJ, in preparation

Stenflo, J. O. 1994, Solar Magnetic Fields: Polarized Radiation Diagnostics (Dordrecht: Kluwer) Stenflo, J. O. 2005, A\&̊A 429, 713

Stenflo, J. O., Keller, C. U., \& Gandorfer, A. 2000, A\&A 355, 789

Trujillo Bueno, J. 1999, in ASSL 243, Solar Polarization, ed. K. N. Nagendra \& J. O. Stenflo, Dordrecht, Kluwer, p.73

Trujillo Bueno, J. 2001, in ASP Conf. Ser. Vol. 236, Advanced Solar Polarimetry: Theory, Observation and Instrumentation, ed. M. Sigwarth (San Francisco: ASP), 161

Trujillo Bueno, J. \& Asensio Ramos, A. 2007, ApJ 655, 642

Trujillo Bueno, J. \& Shchukina, N. 2009, ApJ, in press

Trujillo Bueno, J., Landi Degl'Innocenti, E., Collados, M., Merenda, L., \& Manso Sainz, R. 2002, Nature 415, 403

Trujillo Bueno, J., Shchukina, N., \& Asensio Ramos, A. 2004, Nature 430, 326

\section{Discussion}

BeCKMAn: Could you explain how you use the Hanle effect to distinguish between a tangled field and an aligned field?

Trujillo Bueno: For the case of an aligned field see Trujillo Bueno et al. (2002). For the case of a tangled field see Trujillo Bueno et al. (2004). See also $§ 2.3$.

HeILES: Radio astronomers are familiar with the Goldreich-Kylafis effect. Is this the same as the Hanle effect?

Trujillo Bueno: Since they were considering a hypothetical molecular line with $J_{l}=0$ and $J_{u}=1$ (that is, without the possibility of the lower-level polarization I have discussed in $\S 2.2$ and $\S 2.3$ ) the so-called Goldreich-Kylafis effect is nothing but the upper-level Hanle effect in its saturation limit (because of their additional assumption that the Zeeman splitting is much larger than the natural width of level $J_{u}$.). 